ToKYo J. Math.

VoL. 1, No. 2, 1978

\title{
The Nonvanishing Property of a Weakly Stationary Process
}

\author{
Tatsuo KAWATA \\ Keio University
}

\section{Introduction.}

A measurable function $f(t)$ over $-\infty<t<\infty$ with the following property is said to have the nonvanishing property: if there is a function $g(z), z=t+i y$, analytic on a rectangle $a \leqq t \leqq b, 0<y \leqq r$ (or $a \leqq t \leqq b$, $-r \leqq y<0$ ) and continuous on $a \leqq t \leqq b, 0 \leqq y \leqq r$ (or $a \leqq t \leqq b,-r \leqq y \leqq 0$ ) for some $r>0$ such that $f(t)=g(t)$ almost everywhere in a subinterval interior to $(a, b)$, then $f(t)=g(t)$ should hold almost everywhere throughout $(a, b)$. Such $f(t)$ cannot vanish in any interval unless it is almost everywhere zero over $(-\infty, \infty)$. The situation has been exhaustively studied by Levinson [5], [6], [7], [8]. One of his basic theorems is the following

THEOREM A. Let $f(\lambda)$ be a function of $L^{1}(-\infty, \infty)$ and its Fourier transform be

$$
\widehat{f}(t)=(2 \pi)^{-1 / 2} \int_{-\infty}^{\infty} e^{-i t \lambda} f(\lambda) d \lambda
$$

If

$$
\hat{f}(t)=O(\exp (-\theta(t))), \quad t \rightarrow+\infty,
$$

where $\theta(t)$ is a nondecreasing function over $(c, \infty)$ for some $c>0$ such that

$$
\int_{c}^{\infty} \theta(t) / t^{2} d t=\infty
$$

then $f(\lambda)$ has the nonvanishing property.

Let $(\Omega, \mathscr{F}, P)$ be the given probability space and let $X(t, \omega), \omega \in \Omega$, $-\infty<t<\infty$ be a measurable weakly stationary process with $E X(t, \omega)=0$, 
$-\infty<t<\infty$. Let us denote by $\xi(S, \omega)(S$, Borel set on the real line) and $F(\lambda)$, the spectral random measure and the spectral distribution function of $X(t, \omega)$ respectively. Suppose $F(\lambda)$ satisfies

$$
F(\infty)-F(\lambda)=O\left(e^{-r \lambda}\right), \quad \lambda \rightarrow+\infty
$$

for some $r>0$. Then $X(t, \omega)$ is the boundary of a random function

$$
X(z, \omega)=\int_{-\infty}^{\infty} e^{-i z \lambda} \xi(d \lambda, \omega)
$$

which is well defined and is mean analytic in $-r / 2<\operatorname{Im} z<0$. Furthermore, (1.5) is thought of as a random function which is almost surely analytic as a function of $z$. For details and the proof, see Kawata [4]. As a consequence, the sample functions of $X(t, \omega)$ have the nonvanishing property almost surely.

The purpose of the present paper is to prove Theorem 1 in $\S 1$ which generalizes the above result, appealing to Theorem $A$. In $\S 2$, we shall give the essential part of the proof of the theorem.

The author has discussed the nonvanishing property of a random noise process of shot effect in a vacuum tube [2]. It is shown in $\$ 3$ that the application of Theorem 1 to the random noise process gives the substantial improvement of the result.

\section{§1. Main theorem.}

We are going to prove

TheOREM 1. Suppose $\theta(t)$ is a nondecreasing function over $(c, \infty)$ for some $c>0$ such that (1.3) holds. Then a measurable weakly stationary process $X(t, \omega)$ with the spectral distribution function $F(\lambda)$ satisfying

$$
F(\infty)-F(\lambda)=O(\exp (-\theta(\lambda))), \quad \lambda \rightarrow+\infty
$$

has the nonvanishing property.

Observe first that $X(t, \omega) \exp \left(-t^{2}\right)$ is integrable over $(-\infty, \infty)$ almost surely, which is a consequence of measurability of $X(t, \omega)$ and

$$
\begin{gathered}
E \int_{-\infty}^{\infty}|X(t, \omega)| \exp \left(-t^{2}\right) d t \leqq \int_{-\infty}^{\infty}\left(E|X(t, \omega)|^{2}\right)^{1 / 2} \exp \left(-t^{2}\right) d t \\
\quad=[F(\infty)-F(-\infty)]^{1 / 2} \int_{-\infty}^{\infty} \exp \left(-t^{2}\right) d t<\infty .
\end{gathered}
$$

Then we may define the Fourier transform 


$$
Q(t, \omega)=(2 \pi)^{-1 / 2} \int_{-\infty}^{\infty} X(u, \omega) \exp \left(-u^{2}-i t u\right) d u
$$

almost surely.

Now in order to prove the theorem it is sufficient to show

$$
\sum_{n=1}^{\infty} P\left(\max _{a_{n-1}<t \leqslant a_{n}}|Q(t, \omega)|>\exp \left(-g_{n}\right)\right)<\infty,
$$

where $a_{n}=n^{1 / 4}$ and $g_{n}=\theta\left(a_{n} / 2\right)-3 \log a_{n-1}$. This is seen in the following way. From (2.3), Borel-Cantelli lemma gives us

$$
P\left(\max _{a_{n-1}<t \leqq a_{n}}|Q(t, \omega)|>\exp \left(-g_{n}\right), \text { i.o. }\right)=0 \text {, }
$$

so that there is a set $\Omega_{1}$ with $P\left(\Omega_{1}\right)=1$ such that whenever $\omega \in \Omega_{1}$, $\max _{a_{n-1}<t \leqq a_{n}}|Q(t, \omega)| \leqq \exp \left(-g_{n}\right)$ for $n>N_{0}(\omega)$ for some $N_{0}(\omega)$. Let $t$ be such that $t>a_{N_{0}(\omega)}$. Then there is an $n$ with $a_{n-1}<t \leqq a_{n}$ and we see that for $\omega \in \Omega_{1}$,

$$
|Q(t, \omega)| \leqq \exp \left(-g_{n}\right) \leqq \exp (-g(t)),
$$

where $g(t)=[\theta(t / 2)-3 \log t]$. Since $\int_{0}^{\infty} g(t) / t^{2} d t=\infty$, we see from Theorem A that $X(t, \omega) \exp \left(-t^{2}\right)$ has the nonvanishing property and so does $X(t, \omega)$. This proves the theorem.

Before going to prove (2.3), we note that we may assume, without loss of generality

$$
\theta(t) \leqq t
$$

If not, we may take, in place of $\theta(t)$,

$$
\theta_{1}(t)=\min (\theta(t), t) \text {. }
$$

Obviously $\theta_{1}(t) \leqq t$ and $F(\infty)-F(\lambda)=O\left(\exp \left(-\theta_{1}(\lambda)\right)\right)$ and we may show $\int_{c}^{\infty} \theta_{1}(t) / t^{2} d t=\infty$ (see Levinson, [8] Lemma 19.1, Kawata [3], Lemma 8.3.1).

We also remark that $Q(t, \omega)$ can be written

$$
Q(t, \omega)=2^{-1 / 2} \int_{-\infty}^{\infty} \exp \left(-(t-\lambda)^{2} / 4\right) \xi(d \lambda, \omega),
$$

where, as before $\xi(S, \omega)$ is the random spectral measure of $X(t, \omega)$. Actually

$$
\begin{aligned}
Q(t, \omega) & =(2 \pi)^{-1 / 2} \int_{-\infty}^{\infty} \exp \left(-u^{2}-i t u\right) d u \int_{-\infty}^{\infty} e^{i u \lambda} \xi(d \lambda, \omega) \\
& =(2 \pi)^{-1 / 2} \int_{-\infty}^{\infty} \xi(d \lambda, \omega) \int_{-\infty}^{\infty} \exp \left(-u^{2}-i t u+i \lambda u\right) d u
\end{aligned}
$$


The interchange of the order of integrations here is seen to be permissible if one computes the expectation of the square of the absolute value of the difference of both sides. Since the inner integral is evaluated as $\pi^{1 / 2} \exp \left(-(t-\lambda)^{2} / 2\right),(2.6)$ follows.

$\S 2$. Proof of (2.3).

We proceed to prove the main part (2.3) of the proof of Theorem 1.

Denote the term of the series in $(2.3)$ by $G_{n}$. By the Chebishev inequality, we have

$$
\begin{aligned}
G_{n}= & P\left(\max _{a_{n-1}<t \leq a_{n}}|Q(t, \omega)|>\exp \left(-g_{n}\right)\right) \\
\leqq & \exp \left(2 g_{n}\right) E \max _{a_{n-1}<t \leq a_{n}}|Q(t, \omega)|^{2} \\
= & \exp \left(2 g_{n}\right) E \max _{a_{n-1}<t \leq a_{n}} \mid \int_{-\infty}^{\infty} \exp \left(-\left(\lambda-a_{n}\right)^{2} / 4\right) \\
& \times\left.\exp \left\{\left[\left(\lambda-a_{n}\right)^{2}-(\lambda-t)^{2}\right] / 4\right\} \xi(d \lambda, \omega)\right|^{2} \\
= & \exp \left(2 g_{n}\right) E \max _{a_{n-1}<t \leq a_{n}} \mid \int_{-\infty}^{\infty} \exp \left(-\left(\lambda-a_{n}\right)^{2} / 4\right) \\
& \times\left.\sum_{k=0}^{\infty} 4^{-k}(k !)^{-1}\left[\left(\lambda-a_{n}\right)^{2}-(\lambda-t)^{2}\right]^{k} \xi(d \lambda, \omega)\right|^{2} \\
\leqq & \exp \left(2 g_{n}\right) E \max _{a_{n-1}<t \leq a_{n}}\left[\sum_{k=0}^{\infty} 4^{-k}(k !)^{-1}\left(a_{n}-t\right)^{k}\right. \\
& \left.\times\left|\int_{-\infty}^{\infty} \exp \left(-\left(\lambda-a_{n}\right)^{2} / 4\right)\left(a_{n}+t-2 \lambda\right)^{k} \xi(d \lambda, \omega)\right|\right]^{2} \\
\leqq & \exp \left(2 g_{n}\right) E\left[\sum_{k=0}^{\infty} 4^{-k}(k !)^{-1}\left(a_{n}-a_{n-1}\right)^{k}\right. \\
& \times \max _{a_{n-1}<t \leq a_{n}} \sum_{j=0}^{k}\left(\begin{array}{c}
k \\
j
\end{array}\right)\left(t+a_{n}\right)^{j} \mid \int_{-\infty}^{\infty}(2 \lambda)^{k-j} \exp \left(-\left(\lambda-a_{n}\right)^{2} / 4\right) \\
& \times \xi(d \lambda, \omega) \mid]^{2} \\
\leqq & \exp \left(2 g_{n}\right) E\left[\sum_{k=0}^{\infty} 4^{-k}(k !)^{-1}\left(a_{n}-a_{n-1}\right)^{k}\right. \\
& \left.\times \sum_{j=0}^{k}\left(2 a_{n}\right)^{j}\left(\begin{array}{c}
k \\
j
\end{array}\right)\left|\int_{-\infty}^{\infty}(2 \lambda)^{k-j} \exp \left(-\left(\lambda-a_{n}\right)^{2} / 4\right) \xi(d \lambda, \omega)\right|\right]^{2}
\end{aligned}
$$

which is, by repeated applications of Cauchy inequality,

$$
\begin{aligned}
& \leqq \\
& \quad \exp \left(2 g_{n}\right)\left[\sum _ { k = 0 } ^ { \infty } 4 ^ { - k } ( k ! ) ^ { - 1 } ( a _ { n } - a _ { n - 1 } ) ^ { k } \cdot \sum _ { k = 0 } ^ { \infty } \left\{4^{-k}(k !)^{-1}\left(a_{n}-a_{n-1}\right)^{k}\right.\right. \\
& \left.\quad \times E\left[\sum_{j=0}^{k}\left(2 a_{n}\right)^{j}\left(\begin{array}{c}
k \\
j
\end{array}\right)\left|\int_{-\infty}^{\infty}(2 \lambda)^{k-j} \exp \left(-\left(\lambda-a_{n}\right)^{2} / 4\right) \xi(d \lambda, \omega)\right|\right]^{2}\right\}
\end{aligned}
$$




$$
\begin{aligned}
\leqq & \exp \left(2 g_{n}\right) \exp \left(\left(a_{n}-a_{n-1}\right) / 4\right) \sum_{k=0}^{\infty} 4^{-k}(k !)^{-1}\left(a_{n}-a_{n-1}\right)^{k} \sum_{j=0}^{k}\left(2 a_{n}\right)^{j}\left(\begin{array}{l}
k \\
j
\end{array}\right) \\
& \times E \sum_{j=0}^{k}\left(2 a_{n}\right)^{j}\left(\begin{array}{c}
k \\
j
\end{array}\right)\left|\int_{-\infty}^{\infty}(2 \lambda)^{k-j} \exp \left(-\left(\lambda-a_{n}\right)^{2} / 4\right) \xi(d \lambda, \omega)\right|^{2} \\
= & \exp \left[2 g_{n}+\left(a_{n}-a_{n-1}\right) / 4\right] \\
& \times \sum_{k=0}^{\infty} 4^{-k}(k !)^{-1}\left(a_{n}-a_{n-1}\right)^{k} \sum_{j=0}^{k}\left(2 a_{n}\right)^{j}\left(\begin{array}{c}
k \\
j
\end{array}\right) \cdot \sum_{j=0}^{k}\left(2 a_{n}\right)^{j}\left(\begin{array}{c}
k \\
j
\end{array}\right) \\
& \times \int_{-\infty}^{\infty}(2 \lambda)^{2(k-j)} \exp \left(-\left(\lambda-a_{n}\right)^{2} / 2\right) d F(\lambda) \\
= & \exp \left(2 g_{n}+\left(a_{n}-a_{n-1}\right) / 4\right) \int_{-\infty}^{\infty} \exp \left[\left(a_{n}-a_{n-1}\right)\left(a_{n}+1 / 2\right)\left(a_{n}+2 \lambda^{2}\right)\right] \\
& \quad \times \exp \left(-\left(\lambda-a_{n}\right)^{2} / 2\right) d F(\lambda) .
\end{aligned}
$$

Setting $\left(a_{n}-a_{n-1}\right)\left(a_{n}+1 / 2\right)=p_{n}$ and $\left(a_{n}-a_{n-1}\right)\left(a_{n}+1 / 2\right) a_{n}=q_{n}$, we write the integral in (3.2)

$$
\begin{aligned}
& \left(\int_{-\infty}^{0}+\int_{0}^{a_{n} / 2}+\int_{a_{n} / 2}^{2 a_{n}}+\int_{2 a_{n}}^{\infty}\right) \exp \left(q_{n}+2 p_{n} \lambda^{2}\right) \exp \left(-\left(\lambda-a_{n}\right)^{2} / 2\right) d F(\lambda) \\
& \quad=I_{1}+I_{2}+I_{3}+I_{4}
\end{aligned}
$$

say.

Now we have

$$
\begin{aligned}
I_{1} & =\int_{0}^{\infty} \exp \left[q_{n}+2 p_{n} \lambda^{2}-\left(\lambda+a_{n}\right)^{2} / 2\right] d(1-F(-\lambda)) \\
& \leqq \int_{0}^{\infty} \exp \left(q_{n}+2 p_{n} \lambda^{2}-\lambda^{2} / 2-a_{n}^{2} / 2\right) d(1-F(-\lambda))
\end{aligned}
$$

Here we note that

$$
a_{n}-a_{n-1}=O\left(n^{-3 / 4}\right), \quad p_{n}=O\left(n^{-1 / 2}\right), \quad q_{n}=O\left(n^{-1 / 4}\right) \text {. }
$$

Because of these estimates, $I_{1}$ is seen to be not greater than

$$
\begin{aligned}
\exp & \left(-a_{n}^{2} / 2\right) \cdot \int_{0}^{\infty} \exp \left[(-1 / 2+o(1)) \lambda^{2}\right] d(1-F(-\lambda)) \\
& \leqq \exp \left(-n^{1 / 2} / 2\right) \int_{-\infty}^{0} d F(\lambda) \\
& =C \exp \left(-n^{1 / 2} / 2\right)
\end{aligned}
$$

where $C$ is a constant independent of $n$ larger than some $n_{0}$. As to $I_{2}$, we have

$$
I_{2} \leqq \exp \left(q_{n}+p_{n} a_{n}^{2} / 2-a_{n}^{2} / 8\right) \int_{0}^{a_{n} / 2} d F(\lambda)
$$


which is, because of (3.4),

$$
\begin{aligned}
& \leqq \exp \left[(-1 / 9+o(1)) a_{n}^{2}\right] \int_{0}^{\infty} d F(\lambda) \\
& \leqq C \exp \left(-n^{1 / 2} / 10\right),
\end{aligned}
$$

where $C$ is a constant which may differ from $C$ in (3.5) and the constants that will appear in what follows as well.

We also have

and

$$
\begin{aligned}
I_{3} & \leqq \exp \left(q_{n}+8 p_{n} a_{n}^{2}\right) \int_{a_{n} / 2}^{2 a_{n}} d F(\lambda) \\
& \leqq \exp \left(O\left(n^{-1 / 4}\right)+o(1)\right) \int_{a_{n} / 2}^{\infty} d F(\lambda) \\
& \leqq C \exp \left(-\theta\left(a_{n} / 2\right)\right) \\
& =C \exp \left(-\theta\left(n^{1 / 4} / 2\right)\right)
\end{aligned}
$$

$$
\begin{aligned}
I_{4} & \leqq \int_{2 a_{n}}^{\infty} \exp \left(q_{n}+2 p_{n} \lambda^{2}\right) \exp \left(-\lambda^{2} / 4\right) d F(\lambda) \\
& \leqq \exp \left(q_{n}+\left(8 p_{n}-1\right) a_{n}^{2}\right) \int_{2 a_{n}}^{\infty} d F(\lambda) \\
& \leqq C \int_{2 a_{n}}^{\infty} d F(\lambda) \\
& \leqq C \exp \left(-\theta\left(a_{n} / 2\right)\right) .
\end{aligned}
$$
(2.5),

Altogether we have, from (3.5)-(3.8) and from (3.2), (3.3), noting

$$
\begin{aligned}
G_{n} \leqq & C \exp \left[\theta\left(n^{1 / 4} / 2\right) / 2-(3 / 2) \log (n-1)+C n^{-3 / 4}\right] \\
& \times\left[\exp \left(-n^{1 / 2} / 10\right)+\exp \left(-\theta\left(n^{1 / 4} / 2\right)\right)\right] \\
\leqq & C \exp \left[n^{1 / 4} / 4-(3 / 2) \log (n-1)+C n^{-3 / 4}-n^{1 / 2} / 10\right] \\
& +C \exp [-(3 / 2) \log (n-1)] \\
\leqq & C \exp [-(3 / 2) \log (n-1)]=C(n-1)^{-3 / 2}
\end{aligned}
$$

Hence $\sum G_{n}<\infty$, which shows (2.3). This gives us the proof of Theorem 1.

§3. The nonvanishing of a random noise process.

Let $\left\{U_{n}(\omega), n=0, \pm 1, \cdots\right\}$ be a sequence of independently and identically distributed nonnegative random variables with finite second moments and write 


$$
y(t, \omega)=\sum_{\nu_{n}<t} U_{n}(\omega),
$$

where $\left\{\nu_{n}=\nu_{n}(\omega), n=0, \pm 1, \cdots\right\}$ is a Poisson point process with parameter c. Denote $E U_{n}=\alpha, E U_{n}^{2}=\beta$ and define

$$
Y(t, \omega)=y(t, \omega)-c \alpha t, \quad-\infty<t<\infty
$$

and

$$
X(t, \omega)=\int_{-\infty}^{\infty} \Phi(t-u) d Y(u),
$$

where $\Phi(t)$ is a nonrandom function over $(-\infty, \infty)$ which belongs to $L^{1}(-\infty, \infty) \cap L^{2}(-\infty, \infty)$. The stochastic process $X(t, \omega)$ is called a random noise process (Rice [9], Doob [1], Kawata [2]). This is seen to be a strictly stationary process with finite variance and then a weak stationary process. The corresponding spectral distribution function is given by

$$
F(\lambda)=c \beta \int_{-\infty}^{\lambda}|\phi(u)|^{2} d u,
$$

where $\phi(u)$ is the Fourier transform of $\Phi(t)$ (Kawata [2]).

An immediate consequence of Theorem 1 is

THEOREM 2. Suppose

$$
\int_{\lambda}^{\infty}|\phi(u)|^{2} d u=O(\exp (-\theta(\lambda))), \quad \lambda \rightarrow+\infty,
$$

where $\theta(\lambda)$ is a nondecreasing function over $(c, \infty)$ for some $c>0$, satisfying (1.3). Then the random noise process (4.3) has the nonvanishing property.

This is a refinement of a theorem we have given in [2], in which we placed the condition (1.3) with $\theta(|\lambda|)$ for $|\lambda|<c$ and the condition that $\phi(\lambda)=O(\exp (-\theta(|\lambda|)))$ for large $|\lambda|$ which is stronger than (4.5) because of more conditions we needed there.

\section{References}

[1] J. L. Doob, Stochastic Processes, John Wiley, New York, 1952.

[2] T. Kawata, On the stochastic process of random noise, Ködai Math. Sem. Rep., 7 (1955), 32-42.

[3] T. Kawata, Fourier analysis in probability theory, Acad. Press, New York, 1972.

[4] T. KAwATA, Analytic weakly stationary processes, Keio Engrg. Rep., 28 (1975), 75-86.

[5] N. Levinson, On a theorem of Ingham, J. London Math. Soc., 11 (1936), 103-125. 
[6] N. Levinson, On a class of non-vanishing functions, Proc. London Math. Soc., (2) 41 (1936), 393-407.

[7] N. Levinson, A theorem relating non-vanishing and analytic functions, J. Mathematical Phys., 16 (1938), 185-190.

[8] N. Levinson, Gap and density theorems, Amer. Math. Soc. Colloq. Publ., 26 (1940).

[9] O. S. RICE, Mathematical analysis of random noise, Bell System Tech. J., 23 (1944), 24 (1945), 1-162.

Present Address:

Department of Mathematics

KEIO UNIVERSITY

HIYOSHI-CHO, KOHOKU-KU

YOKOHAMA 223 